\title{
Giuseppe Ricuperati, Frontiere e limiti della ragione. Dalla crisi della coscienza europea all' Illuminismo
}

\section{Wilma Proglio}

\section{(2) OpenEdition}

1 Journals

\section{Edizione digitale}

URL: http://journals.openedition.org/studifrancesi/8907

DOI: 10.4000/studifrancesi.8907

ISSN: 2421-5856

\section{Editore}

Rosenberg \& Sellier

\section{Edizione cartacea}

Data di pubblicazione: 1 octobre 2008

Paginazione: 452-423

ISSN: 0039-2944

\section{Notizia bibliografica digitale}

Wilma Proglio, «Giuseppe Ricuperati, Frontiere e limiti della ragione. Dalla crisi della coscienza europea all'Illuminismo», Studi Francesi [Online], 155 (LII | II) | 2008, online dal 30 novembre 2015, consultato il 08 janvier 2021. URL: http://journals.openedition.org/studifrancesi/8907 ; DOI: https://doi.org/ 10.4000/studifrancesi.8907

Questo documento è stato generato automaticamente il 8 janvier 2021.

\section{(c) $(1) \odot$}

Studi Francesi è distribuita con Licenza Creative Commons Attribuzione - Non commerciale - Non opere derivate 4.0 Internazionale. 


\title{
Giuseppe Ricuperati, Frontiere e limiti della ragione. Dalla crisi della coscienza europea all'Illuminismo
}

\author{
Wilma Proglio
}

\section{NOTIZIA}

GIUSEPPE RICUPERATI, Frontiere e limiti della ragione. Dalla crisi della coscienza europea all'Illuminismo, a cura di Duccio CANESTRI, Torino, Utet, 2006, pp. 379.

1 Come sottolinea Duccio Canestri nella prefazione, l'opera si propone di riunire i saggi di argomento settecentesco che Giuseppe Ricuperati ha scritto tra il 1968 ed il 2003. Tali scritti si collocano in un percorso di rilettura della storia e della storiografia, volto a contribuire ad una nuova ridefinizione del secolo dei Lumi. Nel primo saggio, dal titolo Radicamenti. Cultura italiana e pensiero europeo, l'analisi di Ricuperati parte dalla situazione dell'Italia di fine Seicento, dove per vivacità intellettuale e scambi internazionali si distinguono le città di Roma, Napoli, Firenze e Venezia. Nella prima metà del Settecento si leva la voce riformatrice di Ludovico Antonio Muratori, autore dell'opera Della pubblica felicità. L'abate modenese, portavoce di un modello religioso estraneo al fanatismo ed alla superstizione, ottiene ampi consensi con le sue proposte di rinnovamento dell'istruzione, ispirando le riforme scolastiche di parecchi stati italiani. Milano spicca nel panorama intellettuale del tempo per il periodico Il Caffe e per i personaggi riunitisi intorno a questa realtà editoriale: i fratelli Verri e Cesare Beccaria. Il gruppo, propugnatore di importanti battaglie civili, si ispira da una parte al modello dei giornali morali inglesi e dall'altra all'Encyclopédie.Verso la fine del Settecento si assiste ad un mutamento della geografia intellettuale, con la sempre maggior rilevanza della città di Torino (specialmente grazie all'Accademia delle Scienze) e dei centri minori della Lombardia, interessati dal riformismo di Giuseppe II. Questo è dunque il contesto culturale che accoglie l'eco della Rivoluzione francese: dopo una prima, iniziale reazione positiva da parte dell'opinione pubblica italiana, la 
cattura e l'uccisione del re provocano una netta condanna degli eventi d'oltralpe ed il totale rifiuto della cultura illuministica. Le correnti anti-illuministiche confluiscono nella Restaurazione e nel Romanticismo. A tal proposito Ricuperati mette in guardia dall'uso di categorie quali Preromanticismo e Protoromanticismo, in quanto frutto di un'azione conoscitiva a posteriori, non accompagnata dalla consapevolezza dei protagonisti della vita intellettuale del tempo.

2 Nel saggio successivo, intitolato L'uomo che inventò la crisi della coscienza europea, Ricuperati ricostruisce la vita ed il pensiero di Paul Hazard. Formatosi presso grandi maestri, Brunetière prima e Lanson poi, Hazard esordisce come italianista. Tra le sue opere si ricordano Les premiers contacts des littératures du Nord avec l'esprit latin en Italie, La Révolution française et les lettres italiennes e L'Italie vivante, raccolta di articoli comparsi sulla «Revue des deux mondes». Negli anni Trenta, riflettendo sul contrasto tra la stasi dei paesi europei e la vivacità dell'America, Hazard si sofferma su un altro periodo di crisi vissuto dal vecchio continente, quello compreso tra gli anni 1680-1715, ai quali è seguito un ritrovato equilibrio. La Crise de la conscience européenne individua quattro nuclei tematici. In primo luogo sottolinea l'abbandono della stabilità per l'idea di movimento: mentre un tempo la letteratura si reggeva sui dettami del classicismo, ora le convenzioni saltano e gli studiosi si pongono nei confronti della realtà con l'abito mentale del viaggio e della ricerca. Il secondo nucleo consiste nell'offensiva razionalistica degli eredi di Cartesio. Il terzo focalizza i tentativi di ricostruzione, vale a dire la nascita di nuovi valori e la separazione tra diritto naturale e divino. Il quarto aspetto si configura invece come insorgenza di elementi di "sensiblerie", in un'epoca in cui sembra prevalere la razionalità.

3 Avverso al nazismo (anche se in un primo tempo ammiratore di Mussolini, nel quale vedeva riflessa la forza e la passionalità dello spirito italiano tanto amato da Stendhal), durante la guerra Hazard si fa portavoce di un ideale di cultura europeocentrica, fondato sulla tolleranza e contrapposto alla brutalità e alla violenza. La Pensée européenne au xviiie siècle, uscita postuma nel 1946, individua un'evoluzione del pensiero illuministico articolata in tre tappe: la critica universale, la città degli uomini e la disgregazione, già anticipate nella Crise. In un clima culturale di crescente pessimismo nei confronti delle sorti dell'Europa, la voce di Hazard si distingue per la fede nella possibilità di veder risorgere la grandezza della civiltà occidentale. Lo studio dell'Illuminismo in questo specifico periodo storico si configura proprio come spunto per un cammino di ricostruzione, in un'ottica nuova di engagement, che assegna all'intellettuale una missione pragmatica, calandolo direttamente nelle vicende della storia, assegnandogli un ruolo ed una responsabilità concrete.

4 Nella terza parte, intitolata Crisi della coscienza europea e Illuminismo radicale, Ricuperati si sofferma sulla differenza tra quest'ultimo (di origine anglo-olandese) e l'Illuminismo ufficiale, di stampo prettamente francese, sul quale influiscono notevolmente la massoneria e la cultura dei libertini. Per l'analisi del primo si distingue la figura di Jonathan Israel, studioso della repubblica olandese e degli scambi commerciali tra i vari paesi europei. Anche l'Italia vede la sua fase di Illuminismo radicale precoce, grazie a Doria, Vico, Giannone e Conti.

5 Nel quarto saggio, dal titolo Le categorie di periodizzazione e il Settecento, Ricuperati sottolinea la mancanza di coincidenza tra tempo categoriale e tempo cronologico. Il tentativo di periodizzare un movimento di pensiero implica sempre una ridefinizione non solo del periodo in oggetto, ma anche delle fasi storiche precedenti e successive. 
Nel caso dell'Illuminismo, la riflessione prende avvio a metà Ottocento. La tendenza iniziale è quella di individuare cesure nette tra il secolo dei Lumi e la temperie culturale del secolo successivo. Guizot, Daumiron e Villemain, ad esempio, concordano nel definire il Settecento il trionfo del materialismo e l'Ottocento dello spiritualismo. Per Lemernier l'Illuminismo si identifica nei quattro philosophes per eccellenza: Montesquieu, Voltaire, Diderot e Rousseau. Sempre per Lemernier, il Settecento è specialmente affermazione della coscienza civile, vero motore della Rivoluzione. Avverso alla cultura dei Lumi, Nisard individua invece nel Seicento l'età dell'oro della civiltà francese, salvando del secolo successivo solamente l'esperienza dell'Encyclopédie. Barni ritiene il secolo dei Lumi superiore a quello precedente perché in esso le idee religiose si caratterizzano per essere coronamento e non fondamento di quelle civili. La Déclaration è il documento memorabile del grande rinnovamento ideologico avvenuto nella Francia del Settecento. Analogamente Cassirer, ponendosi in linea di continuità con Kant, vede nell'llluminismo la manifestazione più compiuta di un processo di emancipazione dell'umanità, avviatosi con il Rinascimento. Con il secondo dopoguerra si avverte un nuovo approccio al secolo dei Lumi. Mentre in precedenza si era guardato a quest'ultimo quale fase antecedente e contrapposta al movimento romantico, ora al Settecento viene riconosciuta piena e totale identità e si afferma la tendenza ad esplorarne aspetti meno conosciuti, grazie all'apporto delle scienze umane. Nascono così l'opera di Mauzi sul tema della felicità, quella di Pomeau sulla religione di Voltaire e le opere di Starobinski.

6 Nel quinto saggio Utopia, Settecento, Illuminismo, Ricuperati focalizza la sua attenzione sulle figure di Venturi e Baczko, studiosi uniti dall'esperienza dell'esilio. Venturi, riflettendo sulla differenza tra utopia e riforma, considera la prima nutrimento della seconda e vede il concretizzarsi di questo rapporto nelle culture repubblicane del Settecento. Per Backzo, invece, il nesso tra utopia e riforma è solo uno degli aspetti presi in considerazione in un progetto più ampio di studi in merito alla relazione tra utopia e Lumi.

7 Il sesto saggio, Rileggendo Paul Hazard e Franco Venturi. Gli spazi italiani e la Rivoluzione francese, è dedicato alle reazioni dell'opinione pubblica italiana alle vicende francesi. Il saggio Universalismo e nazione dal Settecento alla Restaurazione tratta l'accezione di alcuni termini quali «universalismo», "patria», «nazione», «straniero», che nel Settecento sono oggetto di una ridefinizione in seguito ai nuovi assetti socio-politici dell'Europa. Vittorio Alfieri (la cui parabola ideologica da «scimmiotto di Voltaire» ad oppositore della cultura francese è paradigmatica di quello che fu l'atteggiamento di molti intellettuali italiani), arriva a concepire un possibile modello di nazione italiana da contrapporre a quello d'oltralpe.

8 Nell'epilogo Ricuperati sottolinea la difficoltà di individuare una data che sigli il tramonto dei Lumi. Egli, in quanto allievo di Venturi, sostiene che in realtà essi non si siano mai esauriti. L'autore individua comunque due date chiave, il 1789 per la Francia e il 1792 per l'Europa, quali imprescindibili punti di svolta. Ricuperati conclude mettendo in evidenza il valore della storia della storiografia, «un modo concreto per ristabilire la relazione con altri lavori e con individualità passate e parallele: un esercizio di rispetto, di comprensione, di pazienza, di tolleranza» (p. 357). 